\title{
AN INTEGRABLE SYSTEM OF PARTIAL DIFFERENTIAL EQUATIONS ON THE SPECIAL LINEAR GROUP
}

\author{
PETER J. VASSILIOU ${ }^{12}$
}

(Received 26 July, 2000; revised 21 May, 2001)

\begin{abstract}
We give an intrinsic construction of a coupled nonlinear system consisting of two firstorder partial differential equations in two dependent and two independent variables which is determined by a hyperbolic structure on the complex special linear group regarded as a real Lie group $G$. Despite the fact that the system is not Darboux semi-integrable at first order, the construction of a family of solutions depending upon two arbitrary functions, each of one variable, is reduced to a system of ordinary differential equations on the 1-jets. The ordinary differential equations in question are of Lie type and associated with $G$.
\end{abstract}

\section{Introduction}

In recent years much attention has focused on studying the solutions of typical partial differential equations (PDEs) of applied mathematics and mathematical physics by reducing them to ordinary differential equations (ODEs) (see for example [8] and references therein). Typically, these reductions to ODEs are only able to describe a "finite-dimensional submanifold" of the "infinite-dimensional manifold" of all the solutions of the PDE. That is, one obtains a family of solutions parametrised by finitely many arbitrary constants rather than a family of solutions parametrised by arbitrary functions. The best known method for carrying out such reductions is by making use of the equation's Lie point symmetry group. ${ }^{3}$

By contrast, if a hyperbolic system consisting of two first-order PDEs in two dependent and two independent variables (say) is Darboux semi-integrable, then it

\footnotetext{
${ }^{\mathrm{I}}$ Centre for Mathematics and its Applications, Australian National University, ACT 0200, Australia; e-mail: pierre@ise.canberra.edu.au.

${ }^{2}$ On leave from the School of Mathematics and Statistics, University of Canberra, ACT, Australia.

(C) Australian Mathematical Society 2002, Serial-fee code 1446-1811/02

${ }^{3}$ However, there is currently a vigorous research program whose aim is to find new reductions beyond those provided by classical Lie methods. Apart from [8], see, for example, the non-exhaustive list $[1,6,7]$, and references therein.
} 
can be shown ([5,9]) that the general solution of the system can be constructed from an ODE system and this ODE system may itself be canonically constructed without having to solve PDE systems. Thus if a hyperbolic first-order system is Darboux semiintegrable, then the construction of its general solution, that is, a family of smooth solutions parametrised by two arbitrary smooth functions each of one variable, is entirely reduced to ODEs.

Roughly speaking, a first-order system is Darboux semi-integrable (at first order) if one of its characteristic systems has at least two independent characteristic invariants ${ }^{4}$ (first-order differential functions which are constant along the system's characteristic flows). An interesting question is the converse: if the construction of the general solution of an (intrinsically) nonlinear hyperbolic system is reducible to ODEs then is the system Darboux semi-integrable? In this paper we establish the existence of a nonlinear, nondegenerate ${ }^{5}$ first-order hyperbolic system, the construction of whose general solution is reduced to an ODE, and yet the system has no regular first-order invariants on either characteristic system. That is, a nonlinear first-order system which is not Darboux semi-integrable at first order but whose general solution can nevertheless be constructed by solving an ODE on the 1-jets. ${ }^{6}$ Another interesting feature of our example arises from the following consideration: it can, in fact, be shown that the constructed system is Darboux semi-integrable at higher order, namely, at order 3. Our construction thus foreshadows the system's integrability at lowest possible order. This begins to address one of the basic open problems in geometric integrability, namely, to find a low order test for Darboux semi-integrability.

We emphasise that, by construction, the ODE system that solves our first-order hyperbolic PDE system is of Lie type, being an ODE on the Lie group $G$, (see [3]). Moreover, the results of this paper have much more general application. Indeed, it follows from our general construction that (almost) every 6-dimensional Lie group gives rise to an integrable PDE system in the class $\mathscr{E}^{1} .^{7}$ Finally, we remark that a number of completely integrable systems, such as the $\mathrm{KdV}$ and the Camassa-Holm equations, are now known to be expressible as (geodesic) flows on certain infinitedimensional Lie groups [2]. It is in this sense that we regard the system constructed in this paper as 'integrable', being, essentially a flow on a finite Lie group.

\footnotetext{
${ }^{4}$ In the literature on hyperbolic first-order systems of the type considered in this paper, characteristic invariants are also called Riemann invariants.

${ }^{5} \mathrm{By}$ 'nondegenerate' we mean that there is no local coordinate system in which the system either completely or partially decouples; see Section 4, especially Proposition 4.1.

${ }^{6}$ Of course, it is not precluded that the system is Darboux semi-integrable at some higher order. Nor is it precluded that, though, as we shall see, the system cannot be linearised by a contact transformation, it may yet have a Bäcklund or Bianchi transform which is ODE solvable. This raises very interesting questions which will not be explored in this paper.

${ }^{7}$ The qualification 'almost' and the restriction to dimension 6 will be apparent from Section 2. However, there is a generalisation which eliminates the dimensional restriction.
} 


\section{Hyperbolic structures and hyperbolic systems}

Our approach to the problem described in the Introduction is via the intrinsic geometry of the class, $\mathscr{E}^{1}$, of first-order systems in two dependent and two independent variables

$$
\bar{F}\left(x, y, u, v, u_{x}, u_{y}, v_{x}, v_{y}\right)=0, \quad \bar{G}\left(\dot{x}, y, u, v, u_{x}, u_{y}, v_{x}, v_{y}\right)=0,
$$

where the matrix

$$
\left(\begin{array}{llll}
\bar{F}_{u_{x}} & \bar{F}_{u_{y}} & \bar{F}_{v_{x}} & \bar{F}_{v_{y}} \\
\bar{G}_{u_{x}} & \bar{G}_{u_{y}} & \bar{G}_{v_{x}} & \bar{G}_{v_{y}}
\end{array}\right)
$$

has rank 2 on the locus $\bar{F}=\bar{G}=0$. This locus defines a 6-dimensional submanifold $\Sigma_{6}$ of the jet bundle $J^{1}\left(\mathbb{R}^{2}, \mathbb{R}^{2}\right)$. The local geometry of (2.1) subject to the above open condition on matrix (2.2) was studied in detail in [11] and shown to be equivalent to the study of certain rank 4 distributions on six-dimensional manifolds. For the convenience of the reader, we recall the pertinent notation. Let $\mathscr{V}$ be a vector field distribution. The subdistribution of characteristic or Cauchy vector fields, Char $\mathscr{V} \subseteq \mathscr{V}$, satisfies [Char $\mathscr{V}, \mathscr{V}] \subseteq \mathscr{V}$. The distribution $\mathscr{V}^{\prime}=\mathscr{V}+[\mathscr{V}, \mathscr{V}]$ is the first derived distribution of $\mathscr{V}$. The higher derived distributions are defined iteratively in an obvious way leading to the derived flag of $\mathscr{V}: \mathscr{V} \subseteq \mathscr{V}^{\prime} \subseteq \mathscr{V}^{\prime \prime} \subseteq \cdots$. The real characteristic variety $[4,9,11]$ of $\mathscr{V}$ is denoted by $\mathscr{C}(\mathscr{V})$.

Let $M_{6}$ be an arbitrary six-dimensional manifold and let $I_{2}$ be a rank 2 Pfaff system on $M_{6}$. The pair $\left(M_{6}, I_{2}\right)$ (or, $\left(M_{6}, I_{2}^{\perp}\right)$ ) is said to be a first-order partial differential equations manifold, if, in a neighbourhood of each point of $M_{6}$ there is a local diffeomorphism $\phi: M_{6} \rightarrow \Sigma_{6}$ such that $\phi^{*} i^{*} \Omega^{1}=I_{2}$, where the functions $\bar{F}, \bar{G}$, defined by $\Sigma_{6}$ satisfy the condition that matrix (2.2) has rank 2 . If such diffeomorphisms exist for $\left(M_{6}, I_{2}\right)$, we say that it is associated with $\left(\Sigma_{6}, i^{*} \Omega^{1}\right)$. In other words, a pair $\left(M_{6}, I_{2}\right)$ is a first-order PDE manifold if $M_{6}$ can be immersed in $J^{1}$ so that $I_{2}$ is a restriction of the contact structure on $J^{1}$ to an equation in $\mathscr{E}^{1}$. We refer to the distribution $\left(i^{*} \Omega^{1}\right)^{\perp}$ as the Vessiot distribution of the associated PDE, [9-11]. For simplicity, we will usually abbreviate first-order PDE manifold to equation manifold.

We now wish to give an intrinsic, geometric characterisation of hyperbolicity for systems in equation class $\mathscr{E}^{1}$, of locally constant type.

DEFINITION 2.1. A rank 4 distribution of vector fields, $H$, on a 6-manifold $M$ that admits a decomposition by rank 2 subdistributions $H_{1}, H_{2}$ satisfying the structure equations

$$
\left[H_{1}, H_{1}\right] \equiv Z_{1}, \quad\left[H_{2}, H_{2}\right] \equiv Z_{2} \quad \bmod H,
$$


is said to be a (rank 4) hyperbolic structure on $M$ and will be denoted by the symbol $H_{1} \oplus H_{2}$. Here the vector fields $Z_{1}, Z_{2}$ are such that $H \cup\left\{Z_{1}, Z_{2}\right\}$ spans $T M$ at each point of $M$.

The following is proved in [11].

THEOREM 2.1. Let $\mathscr{V}$ be a rank 4 distribution on a 6-manifold $M$ such that

(1) $\mathscr{V}$ contains a rank 2 Frobenius integrable subdistribution and

(2) The characteristic variety of $\mathscr{V}$ is a union of projective lines: $\mathscr{C}(\mathscr{V}) \approx \mathbb{R} \mathbb{P}^{1} \cup \mathbb{R} \mathbb{P}^{1}$. Then the pair $\mathscr{M}=(M, \mathscr{V})$ is the equation manifold of a hyperbolic system $\boldsymbol{h} \in \mathscr{E}^{\prime}$ and $\mathscr{V}$ is a hyperbolic structure $H$ on $M$.

We note that though the structure equations of a hyperbolic structure $H$ imply that it has no regular scalar invariants ${ }^{8}$ it may be the case that $H_{1}$ or $H_{2}$ do have such invariants.

DEFINITION 2.2. If $H_{1}$ has $p$ (functionally) independent invariants and $H_{2}$ has $q$, we shall denote this by the symbol $H^{(p, q)}$ and refer to it as a $(p, q)$-hyperbolic structure on $M$.

In accordance with our earlier remarks, we give the following definition.

DEFINITION 2.3. A hyperbolic first-order PDE manifold $\mathscr{M}$ is associated with a Darboux semi-integrable (on the 1-jets) system if $p \geq 2$ or $q \geq 2$; it is associated with a Darboux integrable (on the 1 -jets) system if $p \geq 2$ and $q \geq 2$. . $^{9}$

\section{Hyperbolic structures on the projective group}

In this section we employ Theorem 2.1 to construct some systems of first-order hyperbolic PDEs in two dependent and two independent variables which have the geometric properties we require. We do this by constructing hyperbolic structures on the special linear group $S L(2, \mathbb{C})$ regarded as a real Lie group. From the linear fractional transformations $\mathbb{C} \rightarrow \mathbb{C}$ parametrised by

$$
z \mapsto \frac{a_{1} z+a_{2}}{a_{3} z+1}, \quad\left(\begin{array}{cc}
a_{1} & a_{2} \\
a_{3} & 1
\end{array}\right) \in S L(2, \mathbb{C}),
$$

\footnotetext{
${ }^{8} \mathrm{~A}$ regular scalar invariant of a distribution $\mathscr{V}$ on $M$ is a regular, real-valued function on $M$ that is annihilated by each element of $\mathscr{V}$.

${ }^{9}$ We remark that the list of all systems in the class $\mathscr{E}^{1}$ that are Darboux integrable on the 1-jets has been given in [11].
} 
we obtain the group law

$$
\phi(a, b)=\left(\frac{a_{1} b_{1}+a_{2} b_{3}}{a_{3} b_{1}+1}, \frac{a_{1} b_{2}+a_{2}}{a_{3} b_{1}+1}, \frac{a_{3} b_{1}+b_{3}}{a_{3} b_{1}+1}\right) .
$$

Writing $a_{j}=\alpha_{j}+i \alpha_{j+3}, b_{j}=\beta_{j}+i \beta_{j+3}, j=1,2,3 ; i^{2}=-1$, where $\alpha_{j}, \beta_{j} \in \mathbb{R}$, we obtain the group law for a Lie group $G$ isomorphic to the restricted Lorentz transformations, $S O(3,1, \mathbb{B})^{\dagger}$. Using this group law to compute left- and righttranslations leads to the Lie algebras, $\mathscr{L}$ and $\mathscr{R}$ of, respectively, infinitesimal lefttranslations (right-invariant vector fields) and infinitesimal right-translations (leftinvariant vector fields) on $G$ :

$$
\begin{aligned}
L_{1}= & w_{1} \partial_{w_{1}}+w_{2} \partial_{w_{2}}+w_{4} \partial_{w_{4}}+w_{5} \partial_{w_{5}}, \\
L_{2}= & w_{3} \partial_{w_{1}}+\partial_{w_{2}}+w_{6} \partial_{w_{4}}, \\
L_{3}= & \left(w_{4} w_{5}-w_{1} w_{2}\right) \partial_{w_{1}}+\left(w_{5}^{2}-w_{2}^{2}\right) \partial_{w_{2}}+\left(w_{1}-w_{2} w_{3}+w_{5} w_{6}\right) \partial_{w_{3}} \\
& +\left(w_{2} w_{4}-w_{1} w_{5}\right) \partial_{w_{4}}-2 w_{2} w_{5} \partial_{w_{5}}+\left(w_{4}-w_{3} w_{5}-w_{2} w_{6}\right) \partial_{w_{6}}, \\
L_{4}= & -w_{4} \partial_{w_{1}}-w_{5} \partial_{w_{2}}+w_{1} \partial_{w_{4}}+w_{2} \partial_{w_{5}} \\
L_{5}= & -w_{6} \partial_{w_{1}}+w_{3} \partial_{w_{4}}+\partial_{w_{5}}, \\
L_{6}= & \left(w_{1} w_{5}+w_{2} w_{4}\right) \partial_{w_{1}}+2 w_{2} w_{5} \partial_{w_{2}}-\left(w_{4}-w_{3} w_{5}-w_{2} w_{6}\right) \partial_{w_{3}} \\
& +\left(w_{4} w_{5}-w_{1} w_{2}\right) \partial_{w_{4}}+\left(w_{5}^{2}-w_{2}^{2}\right) \partial_{w_{5}}+\left(w_{1}-w_{2} w_{3}+w_{5} w_{6}\right) \partial_{w_{6}},
\end{aligned}
$$

and

$$
\begin{aligned}
R_{1}= & w_{1} \partial_{w_{1}}+w_{3} \partial_{w_{3}}+w_{4} \partial_{w_{4}}+w_{6} \partial_{w_{6}} \\
R_{2}= & \left(w_{4} w_{6}-w_{1} w_{3}\right) \partial_{w_{1}}+\left(w_{1}-w_{2} w_{3}+w_{5} w_{6}\right) \partial_{w_{2}}+\left(w_{6}^{2}-w_{3}^{2}\right) \partial_{w_{3}} \\
& -\left(w_{3} w_{4}+w_{1} w_{6}\right) \partial_{w_{4}}+\left(w_{4}-w_{2} w_{6}-w_{3} w_{5}\right) \partial_{w_{5}}-2 w_{3} w_{6} \partial_{w_{6}}, \\
R_{3}= & w_{2} \partial_{w_{1}}+\partial_{w_{3}}+w_{5} \partial_{w_{4}}, \\
R_{4}= & -w_{4} \partial_{w_{1}}-w_{6} \partial_{w_{3}}+w_{1} \partial_{w_{4}}+w_{3} \partial_{w_{6}}, \\
R_{5}= & \left(w_{3} w_{4}+w_{1} w_{6}\right) \partial_{w_{1}}-\left(w_{4}-w_{2} w_{6}-w_{3} w_{5}\right) \partial_{w_{2}}+2 w_{3} w_{6} \partial_{w_{3}} \\
& +\left(w_{4} w_{6}-w_{1} w_{3}\right) \partial_{w_{4}}+\left(w_{1}-w_{2} w_{3}+w_{5} w_{6}\right) \partial_{w_{5}}+\left(w_{6}^{2}-w_{3}^{2}\right) \partial_{w_{6}}, \\
R_{6}= & -w_{5} \partial_{w_{1}}+w_{2} \partial_{w_{4}}+\partial_{w_{6}},
\end{aligned}
$$

where $w$ is a local coordinate system around $\mathrm{id}_{G}$ in $G$. Note that Lie algebras $\mathscr{L}$ and $\mathscr{R}$ may be interchanged by the permutation $w_{1} \mapsto w_{1}, w_{2} \mapsto w_{3}, w_{3} \mapsto w_{2}$, $w_{4} \mapsto w_{4}, w_{5} \mapsto w_{6}, w_{6} \mapsto w_{5}$. We record the structure of $\mathscr{L}$ for later use:

$$
\begin{aligned}
& {\left[L_{1}, L_{2}\right]=-L_{2},\left[L_{1}, L_{3}\right]=L_{3}, \quad\left[L_{1}, L_{4}\right]=0, \quad\left[L_{1}, L_{5}\right]=-L_{5},\left[L_{1}, L_{6}\right]=L_{6} \text {, }} \\
& {\left[L_{2}, L_{3}\right]=-2 L_{1},\left[L_{2}, L_{4}\right]=L_{5}, \quad\left[L_{2}, L_{5}\right]=0, \quad\left[L_{2}, L_{6}\right]=-2 L_{4},} \\
& {\left[L_{3}, L_{4}\right]=-L_{6},\left[L_{3}, L_{5}\right]=2 L_{4},\left[L_{3}, L_{6}\right]=0 \text {, }} \\
& {\left[L_{4}, L_{5}\right]=L_{2}, \quad\left[L_{4}, L_{6}\right]=-L_{3} \text {, }} \\
& {\left[L_{5}, L_{6}\right]=2 L_{1} \text {. }}
\end{aligned}
$$


The structure of $\mathscr{R}$ follows from that of $\mathscr{L}$ since $\left[L_{i}, L_{j}\right]=-\left[R_{i}, R_{j}\right]$ for all $i, j$. Using the simply transitive Lie algebras $\mathscr{L}$ and $\mathscr{R}$, we may construct a variety of hyperbolic structures on $G$. Indeed, we claim that

$$
H^{(0,0)}=\left\{L_{2}, L_{6}\right\} \oplus\left\{R_{2}, R_{6}\right\}
$$

is a $(0,0)$-hyperbolic structure on $G$ and that

$$
H^{(0,3)}=\left\{L_{2}, L_{6}\right\} \oplus\left\{R_{3}, R_{6}\right\}
$$

is a $(0,3)$-hyperbolic structure on $G$. For instance, in the case of $H^{(0,0)}=\left\{L_{2}, L_{6}\right\} \oplus$ $\left\{R_{2}, R_{6}\right\}=H_{1} \oplus H_{2}$, we have, using the structure of $\mathscr{L}$ and $\mathscr{R}, H_{1}^{\prime}=\left\{L_{2}, L_{6}, L_{4}\right\}$, $H_{2}^{\prime}=\left\{R_{2}, R_{6}, R_{4}\right\}$. By a MAPLE calculation we verify that $\operatorname{dim} H=4$ and $\operatorname{dim} H^{\prime}=$ 6. Since $[\mathscr{L}, \mathscr{R}]=0$, we deduce that $H^{(0,0)}$ is a hyperbolic structure on $G$. To see that each summand $H_{i}$ has no regular scalar invariants it is easily checked that

$$
\operatorname{dim} H_{i}^{\prime \prime}=5, \quad \operatorname{dim} H^{(k)}=6, \quad k \geq 3 ; \quad i=1,2 .
$$

Similar calculations show that $H^{(0,3)}$ is a $(0,3)$-hyperbolic structure on $G$. An easy calculation shows that the characteristic variety of a hyperbolic structure is the union of two projective lines. By Theorem $2.1, H^{(0,0)}$ and $H^{(0,3)}$ are (locally diffeomorphic to) the Vessiot distributions of first-order hyperbolic systems in two dependent and two independent variables. The system associated with $H^{(0.3)}$ is Darboux semiintegrable having 3 invariants on one of the characteristic systems. As mentioned in the introduction, it can be shown that the local Cauchy problem for this system is solvable by ODEs. This proves that the equation class $\mathscr{E}^{1}$ contains a Darboux semiintegrable system with no regular invariants on one characteristic system and 3 on the other. Other hyperbolic structures on $G$ may be constructed by consideration of the subalgebra classification of $\operatorname{so}(3,1, \mathbb{R})$. Each of these is associated with a hyperbolic first-order system in $\mathscr{E}^{1}$.

\section{An integrable PDE system on the projective group}

For now, however, our interest is in the "least integrable" (from a geometric point of view) of these structures, namely, $H^{(0,0)}$. In reality, the pair $\left(G, H^{(0,0)}\right)$ determines a contact orbit of first-order systems in $\mathscr{E}^{\text {l }}$. Each of these systems is "maximally non-integrable" having no regular scalar invariants on either characteristic system. Despite this, it is not difficult to see that for any fixed system in the contact orbit, a family of solutions, parametrised by two arbitrary functions, each of one variable, may be constructed by ODEs. These ODEs are of Lie type and are associated with the Lie group $G$. We now proceed to the construction of these systems and their solutions. 
By Vessiot's existence theorem [12], it is easy to show that every two dimensional regular integral submanifold ${ }^{10} S \subset G$ of $H$ has tangent space $T_{p} S, p \in S$ so that $T_{p} S$ is spanned by $X_{p}, Y_{p} \in T_{p} S$ such that $X_{p} \in H_{\left.1\right|_{p}}, Y_{p} \in H_{\left.2\right|_{p}}$. Let $f(s)$ and $g(t)$ be real-valued functions defined and smooth on open intervals of the real line $I$ and $J$ respectively, each containing 0 . An inspection of the Lie algebras $\mathscr{L}, \mathscr{R}$ shows that for each $s \in I$ and $t \in J$, the commuting vector fields $L(s)=L_{2}+f(s) L_{6}$ and $R(t)=R_{6}+g(t) R_{2}$ are nonzero elements of $H_{1}$ and $H_{2}$, respectively. Note that $L(s)$ and $R(t)$ are curves in the the Lie algebras of infinitesimal left- and right-translations on $G$. As is well known [3, Lecture 3, Proposition 3], [10, Theorem 4], the initial value problem

$$
\Delta_{*}^{f}\left(\partial_{s}\right)=L(s), \quad \Delta^{f}(0)=x \in G
$$

for a map $\Delta^{f}: I \rightarrow G$, has a unique solution $\Delta^{f}(s)=\lambda_{a(s)}^{f} x$, where $s \mapsto \lambda_{a(s)}^{f}$ is a curve of left-translations in $G$ uniquely determined by the smooth function $f$ and the condition $\lambda_{a(0)}^{f}=\mathrm{id}_{G}$. The initial value problem (4.1) is said to be a system of Lie type associated with the Lie group $G$.

DEFINITION 4.1. Let $\Gamma: g \rightarrow \mathscr{X}(M)$ be a Lie algebra homomorphism from Lie algebra $\boldsymbol{g}$ to the vector fields $\mathscr{X}(M)$ on manifold $M$. Let $A: \mathbb{R} \rightarrow \boldsymbol{g}$ be a curve in $\boldsymbol{g}$. Then the ODE $\gamma_{\star}\left(\partial_{t}\right)=\Gamma(A(t))(\gamma(t)), t \in \mathbb{R}$, for a curve $\gamma: \mathbb{R} \rightarrow M$ is said to be a system of Lie type.

Systems of Lie type were introduced by Lie and Vessiot late in the 19th century and have more recently been studied under the title of ODE systems that admit nonlinear superposition principles [13]. For the case under discussion, the manifold $M$ of Definition 4.1 is the Lie group $G$ and the corresponding Lie algebra is $s o(3,1)$.

Similar to the case of the curve of left-translations, the unique solution of the initial value problem for a map $\Delta^{g}: J \rightarrow G$,

$$
\Delta_{*}^{g}\left(\partial_{t}\right)=R(t), \quad \Delta^{g}(0)=y \in G
$$

is $\Delta^{g}(t)=\rho_{b(t)}^{g} y$, where $t \mapsto \rho_{b(t)}^{g}$ is a curve of right-translations on $G$ uniquely determined by the smooth function $g$ and the condition $\rho_{b(0)}^{g}=\mathrm{id}_{G}$. It follows that

$$
\Delta^{(f, 8)}: I \times J \rightarrow G
$$

defined by

$$
\Delta^{(f, g)}(s, t)=\rho_{b(t)}^{g} \circ \lambda_{a(s)}^{f} x
$$

\footnotetext{
${ }^{10} \mathrm{An}$ immersed submanifold $S \subset G$ is a regular integral submanifold of vector field distribution $\mathscr{V}$ on $G$ if for all $p \in S, T_{p} S \subset \mathscr{V}_{p}$ and $T_{p} S$ is an ordinary integral element $[4,14]$.
} 
is a 2-dimensional regular integral submanifold of $H$ that passes through $x \in G$ and is determined by the two smooth functions $f, g$. Thus we obtain a family of integral submanifolds of $H$ parametrised by the smooth functions $f, g$.

But how is this family of integral submanifolds of distribution $H$ related to solutions of a hyperbolic system in $\mathscr{E}^{1}$ ? In fact, the connection is via Theorem 2.1 which guarantees that $H$ is the Vessiot distribution of some hyperbolic system $\boldsymbol{h}$ in $\mathscr{E}^{1}$. To see this a little more explicitly, note that by a procedure described in [11], the system $\boldsymbol{h}$ may be expressed locally in standard jet bundle coordinates by seeking the four independent invariants of the rank 2 Frobenius distribution $\left\{L_{6}, R_{2}\right\}$. Let $a^{1}, \ldots, a^{4}$ denote its 4 independent invariants and let $\alpha, \beta$ complete these to a local coordinate system on $G$. Introducing these as new coordinates on $G$ transforms distribution $H$ to $\left\{A, B, \partial_{\alpha}, \partial_{\beta}\right\}$, where $A=A^{i}(a, \alpha, \beta) \partial_{a^{i}}, B=B^{i}(a, \alpha, \beta) \partial_{a^{i}}$, for some $A^{i}, B^{i}$. Since $\operatorname{dim} H=4$, it follows that we can diagonalise, and, recycling coefficients and possibly renumbering coordinates present the transformation of $H$ in the form

$$
\left\{A=\partial_{a^{1}}+A^{3} \partial_{a^{3}}+A^{4} \partial_{a^{4}}, B=\partial_{a^{2}}+B^{3} \partial_{a^{3}}+B^{4} \partial_{a^{4}}, \partial_{\alpha}, \partial_{\beta}\right\}
$$

for some $A^{3}, \ldots, B^{4}$. From the structure equations of $H$, it follows easily that Char $H=0$. An argument in [11, Theorem 2.1], then shows that at least one of the 6 Jacobian determinants

$$
\frac{\partial\left(A^{3}, A^{4}\right)}{\partial(\alpha, \beta)}, \frac{\partial\left(A^{3}, B^{3}\right)}{\partial(\alpha, \beta)}, \ldots, \frac{\partial\left(B^{3}, B^{4}\right)}{\partial(\alpha, \beta)}
$$

is not zero. For instance, if the first of these is not zero, then we may make the change of variable $\alpha \mapsto \bar{\alpha}=A^{3}, \beta \mapsto \vec{\beta}=A^{4}$ transforming $H$ to the form

$$
\left\{\partial_{a^{\prime}}+\bar{\alpha} \partial_{a^{3}}+\bar{\beta} \partial_{a^{4}}, \partial_{a^{2}}+B^{3} \partial_{a^{3}}+B^{4} \partial_{a^{4}}, \partial_{\bar{\alpha}}, \partial_{\bar{\beta}}\right\}
$$

We change notation slightly: $a^{1}=x^{1}, a^{2}=x^{2}, a^{3}=u, a^{4}=v, \bar{\alpha}=u_{1}, \bar{\beta}=v_{1}$; $B^{3}=\phi, B^{4}=\psi$ and denote the local diffeomorphism $\left(w_{k}\right) \mapsto\left(x^{1}, x^{2}, u, v, u_{1}, v_{1}\right)$ by $\Psi$. In the new coordinates, $H$ is transformed to $K$, where

$$
K=\left\{\partial_{x_{1}}+u_{1} \partial_{u}+v_{1} \partial_{v}, \partial_{x_{2}}+\phi \partial_{u}+\psi \partial_{v}, \partial_{u_{1}}, \partial_{v_{1}}\right\}
$$

We see that $K=\Psi_{\star} H$ is the Vessiot distribution of the first-order system

$$
\begin{aligned}
& u_{x_{2}}=\phi\left(x_{1}, x_{2}, u, v, u_{x_{1}}, v_{x_{1}}\right) \\
& v_{x_{2}}=\psi\left(x_{1}, x_{2}, u, v, u_{x_{1}}, v_{x_{1}}\right)
\end{aligned}
$$

Being the diffeomorphic image of a hyperbolic structure, $K$ admits a splitting $K=$ $K_{1} \oplus K_{2}$. Now, it is not hard to see that any local diffeomorphism that identifies hyperbolic structures must preserve the corresponding hyperbolic summands and 
hence $\Psi_{\star} H_{i}=K_{i}, i=1,2$. Let $K_{1}$ be spanned by. vector fields $X_{1}, X_{3}$ and $K_{2}$ by $X_{2}, X_{4}$. Thus there are functions $h_{1} \ldots, h_{4}$ such that $\Psi_{\star}\left(L_{2}+f L_{6}\right)=h_{1} X_{1}+$ $h_{3} X_{3}, \Psi_{*}\left(R_{6}+g R_{2}\right)=h_{2} X_{2}+h_{4} X_{4}$. We have $\Psi_{\star}\left\{\partial_{\alpha}, \partial_{\beta}\right\}=\left\{\partial_{u_{1}}, \partial_{v_{1}}\right\}$. A direct calculation, [11, Proposition 3.4] shows that we may take $X_{3}, X_{4} \in\left\{\partial_{u_{1}}, \partial_{v_{1}}\right\}$ and $\left(d x^{1} \wedge d x^{2}\right)\left(X_{1}, X_{2}\right) \neq 0$. We show that $h_{1} h_{2} \neq 0$. Suppose that $h_{1}=0$. Then $\Psi_{\star} L_{2}=h_{3} X_{3}-f \Psi_{\star} L_{6}$ from which one deduces that distribution $\left\{L_{2}, L_{6}\right\}$ has 4 independent invariants, a contradiction. It follows that the integral submanifold $\Psi(S)$ of the Vessiot distribution $K$ has tangent planes which are spanned at each point by the distribution $\left\{X_{1}+\gamma X_{3}, X_{2}+\mu X_{4}\right\}$ for some functions $\gamma, \mu$. It further follows [9, Proposition 2.2] that $\Psi(S)$ is the 1-graph of a solution of the PDE system (4.5). Thus each 2-dimensional regular integral submanifold of $H, \Delta^{(f, g)}$ constructed by solving ODEs of Lie type associated with $G$ is mapped by a local diffeomorphism to a regular integral submanifold of the Vessiot distribution (4.4) which is the 1-graph of a solution of the first-order system (4.5), as we wanted to show. All the constructions in question involve only ODE calculations. An entirely similar argument, mutatis mutandis, gives the desired result for any of the other Jacobian determinants.

Thus we have shown that the general solution of the PDE system determined by the hyperbolic structure $H^{(0,0)}$ on the Lie group $G$ can be constructed by solving only ODEs and this despite the fact that the PDE system is not Darboux semi-integrable (and hence not Darboux integrable) on the 1-jets. Indeed, the PDE system is "maximally non-integrable" on the 1 -jets having no regular characteristic invariants at first order. Furthermore, the ODEs in question are systems of Lie type associated with the Lie group $G$. In a sense, such ODE systems are "nicest possible" being in the class of ODEs (like the elementary Ricatti equation) that admit nonlinear superposition principles.

We conclude this section by remarking on the non-degeneracy of the intrinsically constructed first-order hyperbolic system, $\boldsymbol{h}$. To see that the system is not linearisable by a contact transformation note that it is not hard to show that a hyperbolic system in $\mathscr{E}^{1}$ can be linearised by a contact transformation only if it possesses at least one Riemann invariant on each characteristic system. Another possible source of degeneracy arises from the fact that there may be a coordinate system in which the system either partially or fully decouples. That is, a coordinate system in which the first-order system has the general form

$$
F\left(x, y, u, u_{x}, u_{y}\right)=0, G\left(x, y, u, u_{x}, u_{y}, v, v_{x}, v_{y}\right)=0 .
$$

In this regard we make the following observation.

PROPOSITION 4.1. A hyperbolic system in $\mathscr{E}^{\prime}$ can be transformed to one of the form (4.6) only if at least one of its characteristic systems has 3 independent Riemann invariants of first order. If both of the characteristic systems have 3 independent 
Riemann invariants of first order then the system decouples completely and is in fact contact equivalent to the system $u_{y}=v_{x}=0$.

The proof of the first part, which will be omitted, follows from a case by case analysis of the hyperbolic structure associated with system (4.6). A proof of the second part may be found in [11]. It follows that for our system $\boldsymbol{h}$, no such coordinate system exists. We conclude that $\boldsymbol{h}$ has no local linear representative and is nondegenerate.

\section{Some questions}

We end with some interesting problems. Suppose one is presented with a hyperbolic first-order system in standard jet coordinates, such as (4.5). How can one tell when there are local coordinates on its first-order PDE manifold that reduce it to an ODE of Lie type? That is, how can we tell when a first-order hyperbolic system is an ODE on a Lie group? In this paper, we have not directly addressed this important issue. If this could be carried out by ODE methods alone, it would decisively answer the question posed in the Introduction on Darboux semi-integrability and ODE solvability of a hyperbolic first-order system. Another problem is to explore the possible linearisation of the system $\boldsymbol{h}$ by Bianchi-type transformations via the well-known linearisation of ODE systems of Lie type. Finally, construct the system explicitly in standard coordinates and give a clean proof that every, say local $C^{1}$, solution can be constructed by ODEs of Lie type. Progress with these and related questions will be reported elsewhere.

\section{References}

[1] I. M. Anderson, M. E. Fels and C. G. Torre. "Group invariant solutions without transversality", Commun. Math. Phys. 212 (2000) 653-686.

[2] V. I. Arnold and B. A. Khesin, Topological Methods in Hydrodynamics (Springer, 1998).

[3] R. L. Bryant, "An introduction to Lie groups and symplectic geometry", in Geometry and quantum field theory (Park City, UT, 1991), IAS/Park City Math. Ser. 1, (Amer. Math. Soc., Providence, RI, 1995) 5-181.

[4] R. L. Bryant, S. S. Chern, R. B. Gardner, H. L. Goldschmidt and P. A. Griffiths, Exterior Differential Systems, Mathematical Sciences Research Institute Publications 18 (Springer, New York, 1991).

[5] R. L. Bryant, P. A. Griffiths and L. Hsu, "Hyperbolic exterior differential systems and their conservation laws I, II", Selecta Math. (N.S.) 1 (1995) 21-112, 265-323.

[6] P. W. Doyle, "Characteristic solutions of scalar Newton equations in one space dimension", Internat. J. Non-Linear Mech. 33 (1998) 1013-1026.

[7] P. W. Doyle, "Symmetry and ordinary differential constraints", Internat. J. Non-Linear Mech. 34 (1999) 1089-1102. 
[8] M. C. Nucci, "The role of symmetries in solving differential equations", Mathl. Comput. Modelling 25 (1997) 181-193.

[9] P. J. Vassiliou, "Vessiot structure for manifolds of $(p, q)$-hyperbolic type: Darboux integrability and symmetry", Trans. Amer. Math. Soc. 353 (2001) 1705-1739.

[10] P. J. Vassiliou, "Tangential characteristic symmetries and first order hyperbolic systems", Special issue "Computational geometry for differential equations", Appl. Algebra Engrg. Comm. Comput 11 (2001) 377-395.

[11] P. J. Vassiliou, "Intrinsic geometry of first order partial differential equations in the plane", Research report \#2000/4, School of Mathematics and Statistics, University of Canberra, http: //beth. canberra.edu.au/peterv.

[12] E. Vessiot, "Sur une théorie nouvelles des problèmes généraux d'intégration", Bull. Soc. Math. France 52 (1924) 336-395.

[13] P. Winternitz, "Lie groups and solutions of differential equations", in Nonlinear phenomena (Oaxtepec, 1982), Lecture Notes in Phys. 189, (Springer, Berlin, 1983) 263-331.

[14] K. Yang, Elementary Exterior Differential Systems and its Applications to Equivalence Problems (Kluwer, 1992). 